\title{
Determination of Energy-Transfer Distributions in Ionizing Ion-Molecule Collisions
}

\author{
S. Maclot,,${ }^{1, *}$ R. Delaunay, ${ }^{1}$ D. G. Piekarski, ${ }^{2}$ A. Domaracka, ${ }^{1}$ B. A. Huber,${ }^{1}$ L. Adoui, ${ }^{1}$ F. Martín, $, 3,4$ \\ M. Alcamí, ${ }^{2,3}$ L. Avaldi, ${ }^{5}$ P. Bolognesi, ${ }^{5}$ S. Díaz-Tendero, ${ }^{2,4}$ and P. Rousseau ${ }^{1, \dagger}$ \\ ${ }^{1}$ Normandie Université-CIMAP, UMR 6252 CEA/CNRS/ENSICAEN/UNICAEN, \\ Boulevard Henri Becquerel, BP 5133-14070 Caen cedex 5, France \\ ${ }^{2}$ Departamento de Química, Módulo 13, Universidad Autónoma de Madrid, 28049 Madrid, Spain \\ ${ }^{3}$ Instituto Madrileño de Estudios Avanzados en Nanociencias (IMDEANanociencia), Cantoblanco 28049 Madrid, Spain \\ ${ }^{4}$ Condensed Matter Physics Center (IFIMAC), Universidad Autónoma de Madrid, 28049 Madrid, Spain \\ ${ }^{5}$ CNR-Istituto di Struttura della Materia, Area della Ricerca di Roma 1, Monterotondo Scalo, Italy
}

(Received 29 January 2016; published 8 August 2016)

\begin{abstract}
The ionization and fragmentation of the nucleoside thymidine in the gas phase has been investigated by combining ion collision with state-selected photoionization experiments and quantum chemistry calculations. The comparison between the mass spectra measured in both types of experiments allows us to accurately determine the distribution of the energy deposited in the ionized molecule as a result of the collision. The relation of two experimental techniques and theory shows a strong correlation between the excited states of the ionized molecule with the computed dissociation pathways, as well as with charge localization or delocalization.
\end{abstract}

DOI: 10.1103/PhysRevLett.117.073201

The understanding of the electronic and nuclear dynamics in molecular systems induced by sudden ionization or excitation, which drives chemical reactions, offers new opportunities for controlled ultrafast chemistry [1]. For example, the charge migration on the fs time scale after hole formation, which triggers atomic motion and molecular fragmentation [2] or the localization of multiple charges in specific molecular groups and the subsequent Coulomb explosion has recently been observed $[3,4]$. Ultrafast nuclear rearrangements have been also observed in pump-probe experiments [5]; such processes compete with the expected charge separation in multiply charged molecules [6-8]. Therefore, a detailed knowledge of the response of complex molecular systems to ionization or excitation and its influence on chemical reactivity is still a relevant topic today $[9,10]$. In this context, recent combined experimental and theoretical works have been very valuable in providing pictures of the ion-induced ionization or fragmentation of complex molecular systems [7,8,11,12]. However, a meaningful comparison between experimental and theoretical results requires knowledge of the energy transferred in the collision, which is in fact represented by a wide energy distribution due to interactions at different impact parameters. Pioneering experimental work reported in Refs. [13,14] has already been performed in order to determine the actual energy-deposit distributions in ionmolecule collisions, as well as to study its relationship with the observed fragmentation patterns. However, these methods require the knowledge of the initial and final projectile states which is only straightforward in the case of doubleelectron capture by singly charged ions (e.g., $\mathrm{H}^{+} \rightarrow \mathrm{H}^{-}$), which is more the exception than the rule.
Here we report on the ionization and fragmentation of a DNA building block, the nucleoside thymidine combining (i) ion collisions, (ii) VUV photoionization, along with (iii) $a b$ initio calculations. Combining such state-ofthe-art techniques, we provide a complete picture of the charge localization and the excitation energy distribution in complex molecular systems after interaction with ionizing radiation. More importantly, it becomes possible to determine the energy deposited in the target as a result of an ionizing collision with ions, which is the primary process associated with radiation damage. With the development of cancer therapies based on ionizing particles, such as hadrontherapy [15], a better understanding of the radiation damage via a multiscale and multidisciplinary approach has become unavoidable [16]. At the molecular scale, this relies on the investigation of ionization or fragmentation of molecules of biological interest in the gas phase at different energy ranges [11,17-19].

The experiments have been performed at ARIBE, the low-energy ion facility of GANIL (Caen, France) and at the GASPHASE beam line of the synchrotron radiation facility ELETTRA (Trieste, Italy). Both experiments are based on crossed-beam setups using coincidence time-of-flight mass spectrometry. The photoionization experiments are based on state-selected mass spectrometry using photoelectronphotoion coincidence measurements (PEPICO). The effusive beam of the neutral thymidine molecule [2'-deoxythymidine abbreviated dThy; see structural formula in Fig. 1(a)] was produced by heating a powder in an oven at a temperature low enough to avoid thermal decomposition [20]. Both experimental setups have been described 


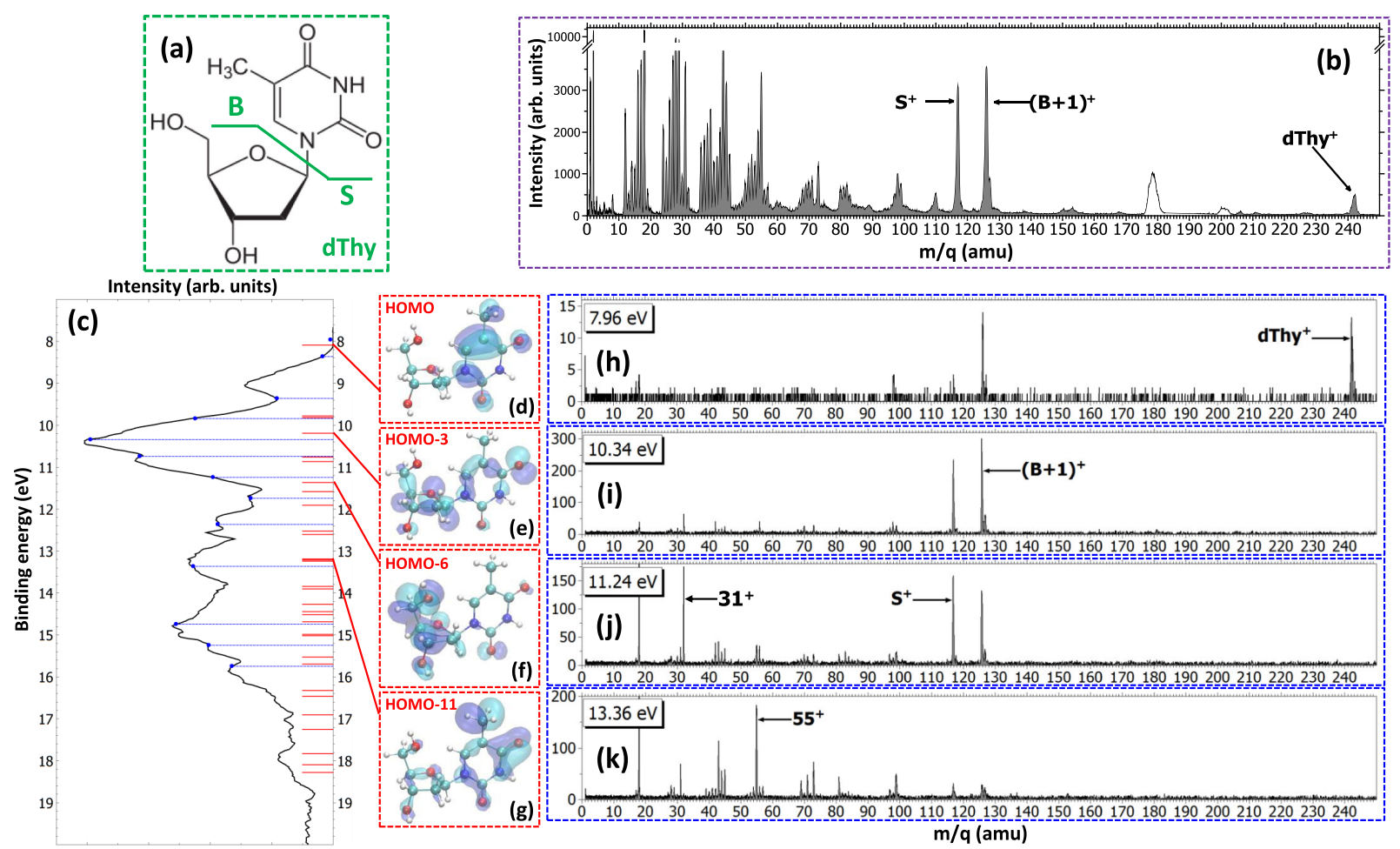

FIG. 1. (a) Structural formula of thymidine. Considering the glycosidic bond cleavage, the fragments produced are noted B and S for the base and sugar parts, respectively. (b) Mass spectrum of thymidine after the ionization by $48 \mathrm{keV} \mathrm{O}^{6+}$ ions. White peaks around $m / q=180$ and 200 amu are due to pollutions. (c) Photoelectron spectrum (PES) of thymidine obtained at $50 \mathrm{eV}$ (black curve). The blue dashed lines show the energy values chosen for PEPICO measurements. Red bars correspond to orbital energy values computed with the OVGF method. Panels (d)-(g) show the electron density of different molecular orbitals. Panels (h)-(k) show the PEPICO mass spectra recorded for different binding energies of the electron corresponding to closest energies to the orbitals presented.

in detail elsewhere [21,22] and a brief description is given in the Supplemental Material [23].

In order to have a picture of the stability of the charged thymidine in the gas phase, the mass spectrum of the charged products detected after the production of singly charged thymidine in the interaction with $48 \mathrm{keV} \mathrm{O}^{6+}$ ions is shown in Fig. 1(b). The peak located at $m / q=242 \mathrm{amu}$ corresponds to the intact singly charged $\mathrm{dThy}^{+}$and shows that a fraction of the parent population can be stable, at least in the $\mu$ s time scale, after single ionization. The main peaks among the heaviest fragments are observed at $m / q=117$ and $126 \mathrm{amu}$. The first one corresponds to the sugar part $\mathrm{S}^{+}$, whereas the second one is assigned to the fragment $(\mathrm{B}+1)^{+}$corresponding to an intramolecular rearrangement associated with a hydrogen transfer to the base part B [24]. Both fragments are the result of the glycosidic bond cleavage, an important mechanism in the radiation damage of DNA [24-26], and contribute to 8\% of the spectrum. A very small amount of fragments heavier than the base or sugar parts, i.e., loss of neutral fragments keeping intact the glycosidic bond, are also observed. This is due to the large distribution of impact parameters in the case of ion collisions [27], which leads to energy transfers, spanning from a few $\mathrm{meV}$ to few tens of $\mathrm{eV}$ and involves a distribution of vibrational energy transfer and electron captures in various electronic states. Thus, the knowledge of the distribution of the energy transferred to the molecule plays a key role to unravel its fragmentation dynamics. It is difficult to assess experimentally this energy distribution even if translational spectroscopy can provide it in the case of multiple electron capture $[13,14]$.

A method that can provide direct insight on the fragmentation following a selected energy deposition is the PEPICO technique, where the kinetic energy of the photoelectrons allows us to pinpoint the energy left in the target [28]. The photoelectron spectrum of thymidine measured at $50 \mathrm{eV}$ is shown in Fig. 1(c). From this spectrum, thirteen photoelectron binding energy values $E_{b}$, covering the main features, have been chosen to study the evolution of the fragmentation. A simulation of the photoelectron binding energy spectra was carried out by computing the ionization energies for the 31st highest molecular orbitals using the outer-valence Green's function (OVGF) method [29] in combination with a $6-311 \mathrm{G}(d, p)$ basis set of the GAUSSIAN09 package [30]. The results are plotted in panel (c). This method incorporates the effects of electron correlation in the computation of molecular ionization potentials as one-particle theory for the description of ultrafast electron charge density dynamics after ionization of an outer-valence electron. The uncertainty of the 
calculated energies by this method is about $\pm 0.3 \mathrm{eV}$ [31]. Figures $1(\mathrm{~d})-1(\mathrm{~g})$ show the computed electron densities of four orbitals corresponding to the HOMO, HOMO - 3, HOMO -6 , and HOMO -11 with binding energies of $8.09,10.19,11.36$, and $13.18 \mathrm{eV}$, respectively, which are the closest in energy to the four selected PEPICO mass spectra shown in Figs. 1(h)-1(k). They illustrate that the charge localization after ionization strongly depends on the orbital and may lead to different fragmentation channels. In the first mass spectrum recorded at $E_{b}=7.96 \mathrm{eV}$ [Fig. 1(h)] we observe the peak due to the parent ion, i.e., the singly charged thymidine molecule. Because of the experimental energy resolution in the PEPICO measurements, not only photoelectrons from the ground ionic state but also from deeper orbitals can be detected [23]. This may lead to molecular dissociation and indeed some fragments are also observed. The main fragment corresponds to $(\mathrm{B}+1)^{+}$, indicating that the charge is mainly located on the base part as suggested by the electronic density of the HOMO [panel (d)]. In the next mass spectrum, measured at $E_{b}=10.34 \mathrm{eV}$, two main peaks are observed with similar intensities. They are assigned to the fragment $(\mathrm{B}+1)^{+}$and $\mathrm{S}^{+}$as observed in the case of the fragmentation induced by multiply charged ions. The similar intensities show that the charge has almost the same probability to be located on each one of the two moieties of the molecule as shown by the nonpreferential charge localization in the orbital HOMO - 3, in panel (e), and neighboring orbitals [23]. Panel (j) presents the mass spectrum recorded at $E_{b}=11.24 \mathrm{eV}$. The same main peaks are present, but fragment $\mathrm{S}^{+}$is now prominent. This is consistent with the preferential charge localization of the associated orbital HOMO - 6 [panel (f)], although the neighboring orbitals can contribute to $\mathrm{S}^{+}$and $(\mathrm{B}+1)^{+}$peaks [23]. At larger excitation energy [Fig. 1(k)], the mass spectrum is characterized by a strong fragmentation showing a redistribution of the transferred energy leading to the cleavage of several bonds in the molecule.

Using the energy selected PEPICO mass spectra we can evaluate the excitation energy distribution in an ion collision. This is achieved by fitting the results of the PEPICO spectra via a constrained linear least-square regression to the ion-induced mass spectrum considering eleven most relevant features. The fit parameters represent the contribution of each PEPICO mass spectrum, i.e., the contribution of the fragmentation of a bunch of excited states of the singly charged ion, to the ion spectrum (see method in the Supplemental Material [23]). The result is displayed in Fig. 2 as a function of the excitation energy defined as the difference between the energy left in the target and the ionization potential. The energy distribution increases smoothly up to a maximum around $2-3 \mathrm{eV}$ and then it extends up to $8 \mathrm{eV}$ and likely also above this energy, in a region not investigated in the present PEPICO experiments. Collisions at closer impact parameters can explain the extended tail towards larger deposited energy [27,32,33]. Penetrating trajectories are associated with large deposit energy of several tens of $\mathrm{eV}$ [33]. However, in the present interaction of $48 \mathrm{keV} \mathrm{O}^{6+}$ with thymidine, peripheral collisions leading to small energy transfer are dominating. This is due to the fact that the electron capture radius is large ( 20 a.u. considering the classical-over-barrier model [34]) compared to the molecular size ("radius" of $\sim 8$ a.u.). The form of the distribution shown in Fig. 2 is qualitatively similar to those obtained by fitting theoretical fragmentation probabilities to experimental measured branching ratios in small carbon clusters [27] and fullerenes [33,35], in which the energy distribution was the fitting parameter, thus showing that the present results are also compatible with previous empirical estimations. Notice that, although the set of accessible target states can, in principle, be different in photoionization and collision processes, due to the different conservation rules that can apply in each case, this is not a problem in the present work because the absence of any symmetry in the molecular target does not restrict the number of accessible states in either process. Moreover, the single-electron capture, which is the dominant process at impact energies considered in this work, is not accompanied by excitation of target and projectile electrons [33,35]. Therefore, one can safely assume that the mass spectra resulting from the collision involves the same target states as the PEPICO spectra.

According to the PEPICO results, the maximum in the distribution of excitation energy corresponds to the region of the HOMO - 3 state. The charge distribution [Fig. 1(e)] leads to the cleavage of the glycosidic bond and the production of both $(\mathrm{B}+1)^{+}$and $\mathrm{S}^{+}$fragments as observed in ion-collision. Larger excitation energy will cause further fragmentation. The partial ion yields of the parent ion and the leading fragments in the PEPICO mass spectra are plotted as a function of the binding energy $E_{b}$ in Fig. 3. The parent ion has a maximum yield centered around $8.5 \mathrm{eV}$ and

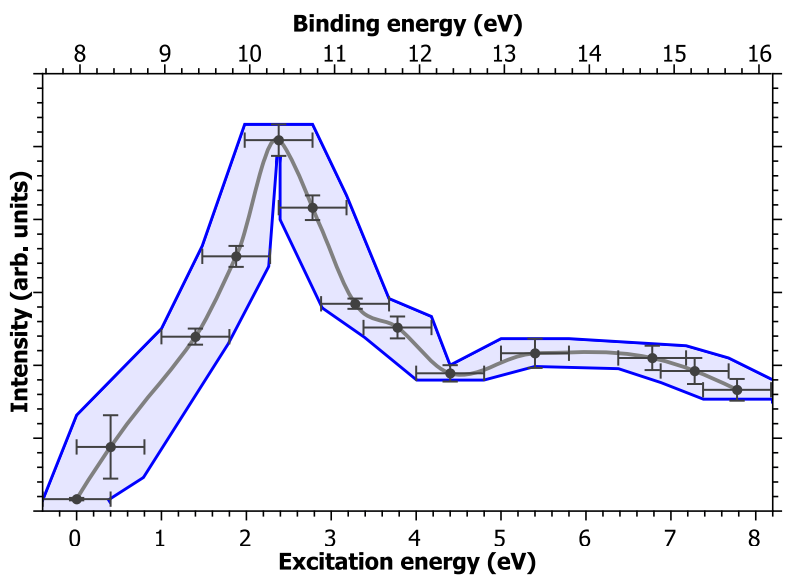

FIG. 2. Determined distribution of the excitation energy in the ion collision (see text). The $R^{2}$ coefficient of determination for this fit is 0.86 . 

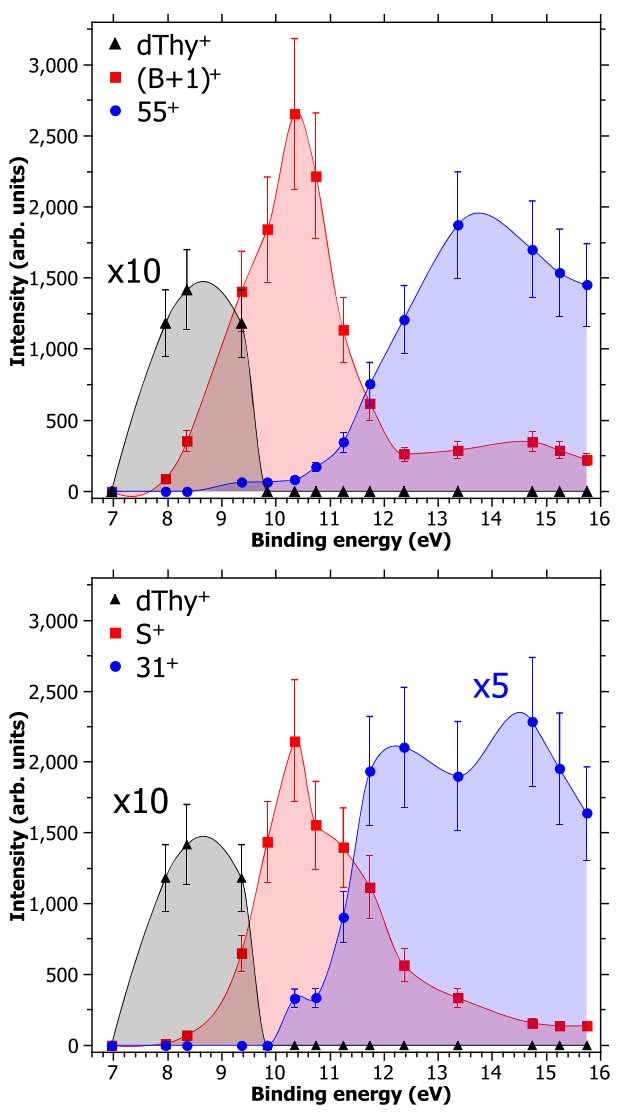

FIG. 3. Partial ion yields of some products as a function of the binding energy obtained from PEPICO. (a) Ionized thymidine $\left(\mathrm{dThy}^{+}\right)$and base fragments $\left[(\mathrm{B}+1)^{+}\right.$and $\left.55^{+}\right]$. (b) Ionized thymidine and sugar fragments $\left(\mathrm{S}^{+}\right.$and $\left.31^{+}\right)$. Each point of the curves represents the areas of a Gaussian fit to the mass spectra peaks. Error bars are estimated to $20 \%$ of the value due to the fitting method. then vanishes above $10 \mathrm{eV}$, while the partial yields of the main fragments $(\mathrm{B}+1)^{+}$and $\mathrm{S}^{+}$are observed over a wide $E_{b}$ range starting around $8.5 \mathrm{eV}$ and display maximum around $10 \mathrm{eV}$ (Fig. 3). Secondary dissociation of these fragments is observed for higher $E_{b}$ which corresponds to the tail towards larger excitation energies in Fig. 2. Fragments at $m / q=55 \mathrm{amu}$ have been previously assigned to $\mathrm{C}_{3} \mathrm{H}_{3} \mathrm{O}^{+}$arising from the base part [24]. Several pathways leading to this fragment have been calculated, as shown in Fig. 4. The quantum chemistry calculations for the secondary fragmentation rely on an exploration of the potential energy surface in the ground state, i.e., assuming fast redistribution of the excitation energy over the vibrational degrees of freedom. The structure of the neutral molecule in the gas phase, its ionic form, and the fragments produced in the relevant exit channels, together with the associated dissociation energy, have been computed with the density functional theory at the B3LYP/6-31G $(d, p)$ level of theory, using GaUssian09 (B3LYP denotes the Becke three-parameter Lee-Yang-Parr hybrid functional) [30]. The simulations show that fragment $\mathrm{C}_{3} \mathrm{H}_{3} \mathrm{O}^{+}$can be produced from the base part $\mathrm{B}^{+}$ [panel (b)] and from $(\mathrm{B}+1)^{+}$[panel (c)], but also from the sugar part $\mathrm{S}^{+}$[panel (b)]. More surprisingly, it is also possible to form this fragment directly from $\mathrm{dThy}^{+}$without glycosidic bond breaking [panel (d)]. The second fragment at $m / q=31$ is assigned to $\mathrm{HOCH}_{2}^{+}$. This fragment arises from the sugar part [25] and certainly from the outside part of the furanose ring [32]. Computed formation mechanisms show that it is possible to obtain this fragment from the sugar part while keeping intact the glycosidic bond [Figs. 4(b) and 4(d), respectively]. Thus, combining the partial ion yields measured in the PEPICO experiments, the calculation of the binding energies of the different ionic

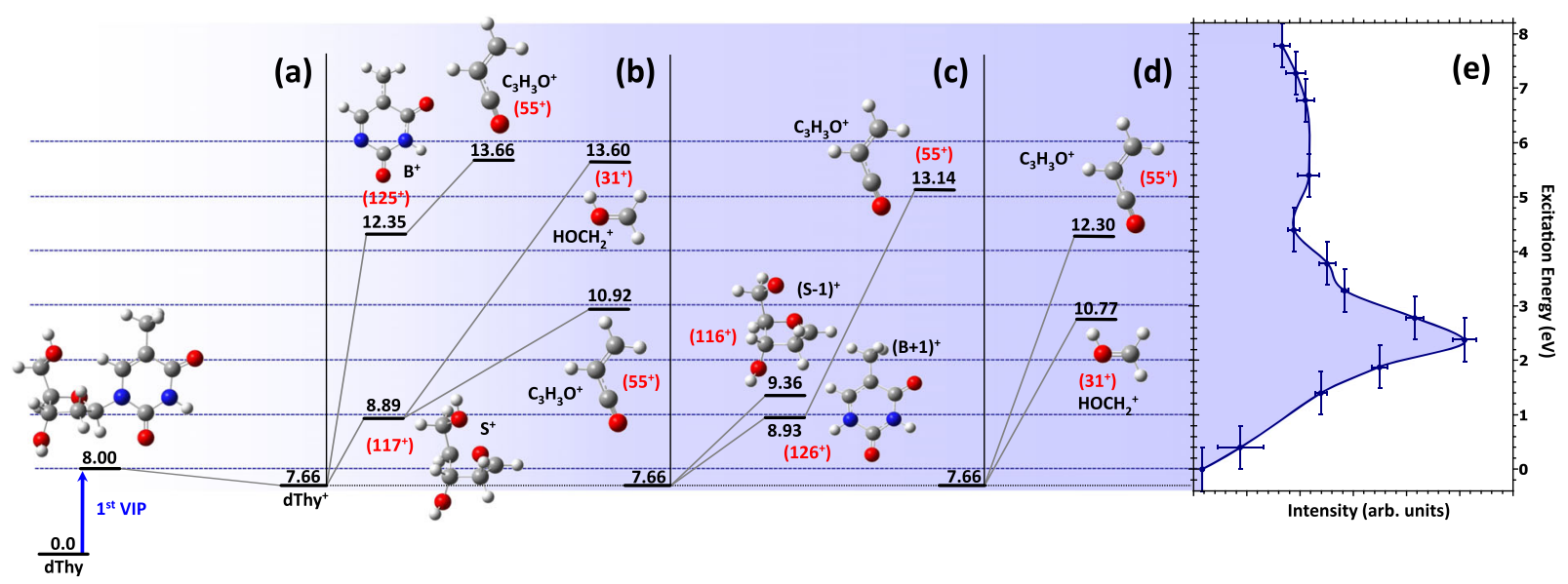

FIG. 4. (a) First vertical ionization potential and the most stable conformer of the singly charged thymidine (dThy $\left.{ }^{+}\right)$. (b), (c) and (d) Energy levels of some fragmentation pathways obtained after the exploration of the potential energy surface of the most stable conformer of the singly charged thymidine. The calculated barriers of all pathways are not shown here for the sake of clarity. (e) Determined distribution of excitation energy. 
states with the OVGF method and the dissociation pathways one can evaluate the contribution of the different fragmentation channels to the distribution of the energy transfer in the ion collision.

In summary, this Letter presents the proof of concept of a method to accurately determine the excitation energy distribution of complex molecular ions produced in collisions with fast ions without knowledge of the initial and final states of the projectile nor the ionization potential of the target. The method relies on the combination of photon and ion experiments. The additional support of quantum chemistry calculations allows us to rationalize the measured energy distributions in terms of the electronic states of the singly charged ion and fragmentation channels. Thus, the combination of ion and electron velocity resolved spectroscopies with in situ photoionization experiments appears as a promising tool to obtain a complete picture of the molecular dynamics that follows a collision with fast ions.

We thank F. Noury, S. Guillous, R. Richter, M. Coreno, and K. Prince for support as well as L. J. Avaldi for advice in the statistical analysis of the data. We acknowledge the allocation of computer time at the Centro de Computación Científica at the Universidad Autónoma de Madrid (CCCUAM). This work was partially supported by the projects FIS2013-42002-R and CTQ2013-43698-P (MINECO), NANOFRONTMAG (CAM), PHC Galilée 28137PE, and an Advanced Grant of the European Research Council XCHEM No. 290853. Research was conducted in the framework of the International Associated Laboratory (LIA) "Fragmentation dynamics of complex molecular systems-DYNAMO" and in the COST actions XLIC (CM1204) and Nano-IBCT (MP1002). D. G. P. acknowledges the FPI grant of the Universidad Autónoma de Madrid. S. D.-T. gratefully acknowledges the "Ramón y Cajal" program of the Spanish Ministerio de Educación y Ciencia.

*sylvain.maclot@fysik.lth.se † patrick.rousseau@ganil.fr

[1] Ultrafast Phenomena in Molecular Sciences, edited by R. de Nalda and L. Bañares, Springer Series in Chemical Physics, (Springer Intl., Heidelberg, New York, Dordrecht, London, 2014), Vol. 107.

[2] F. Calegari, D. Ayuso, A. Trabattoni, L. Belshaw, S. De Camillis, S. Anumula, F. Frassetto, L. Poletto, A. Palacios, P. Decleva, J. B. Greenwood, F. Martín, and M. Nisoli, Science 346, 336 (2014).

[3] B. Erk et al., Phys. Rev. Lett. 110, 053003 (2013).

[4] A. N. Markevitch, D. A. Romanov, S. M. Smith, and R. J. Levis, Phys. Rev. Lett. 92, 063001 (2004).

[5] Y. H. Jiang, A. Rudenko, O. Herrwerth, L. Foucar, M. Kurka, K. U. Kühnel, M. Lezius, M. F. Kling, J. van Tilborg, A. Belkacem, K. Ueda, S. Düsterer, R. Treusch, C. Schröter, R. Moshammer, and J. Ullrich, Phys. Rev. Lett. 105, 263002 (2010).
[6] S. De, J. Rajput, A. Roy, P. N. Ghosh, and C. P. Safvan, Phys. Rev. Lett. 97, 213201 (2006).

[7] S. Maclot, D. G. Piekarski, A. Domaracka, A. Méry, V. Vizcaino, L. Adoui, F. Martín, M. Alcamí, B. A. Huber, P. Rousseau, and S. Díaz-Tendero, J. Phys. Chem. Lett. 4, 3903 (2013).

[8] D. G. Piekarski, R. Delaunay, S. Maclot, L. Adoui, F. Martín, M. Alcamí, B. A. Huber, P. Rousseau, A. Domaracka, and S. Díaz-Tendero, Phys. Chem. Chem. Phys. 17, 16767 (2015).

[9] L. Levin, W. Skomorowski, L. Rybak, R. Kosloff, C. P. Koch, and Z. Amitay, Phys. Rev. Lett. 114, 233003 (2015).

[10] Z. Li, O. Vendrell, and R. Santra, Phys. Rev. Lett. 115, 143002 (2015).

[11] P. López-Tarifa, M.-A. Hervé du Penhoat, R. Vuilleumier, M.-P. Gaigeot, I. Tavernelli, A. Le Padellec, J.-P. Champeaux, M. Alcamí, P. Moretto-Capelle, F. Martín, and M.-F. Politis, Phys. Rev. Lett. 107, 023202 (2011).

[12] M. Capron, S. Díaz-Tendero, S. Maclot, A. Domaracka, E. Lattouf, A. Ławicki, R. Maisonny, J.-Y. Chesnel, A. Méry, J.-C. Poully, J. Rangama, L. Adoui, F. Martín, M. Alcamí, P. Rousseau, and B. A. Huber, Chem. Eur. J. 18, 9321 (2012).

[13] L. Chen, S. Martin, J. Bernard, and R. Brédy, Phys. Rev. Lett. 98, 193401 (2007).

[14] R. Brédy, J. Bernard, L. Chen, G. Montagne, B. Li, and S. Martin, J. Chem. Phys. 130, 114305 (2009).

[15] M. Durante and J. S. Loeffler, Nat. Rev. Clin. Oncol. 7, 37 (2010).

[16] A. V. Solov'yov, E. Surdutovich, E. Scifoni, I. Mishustin, and W. Greiner, Phys. Rev. E 79, 011909 (2009).

[17] B. Liu, S. B. Nielsen, P. Hvelplund, H. Zettergren, H. Cederquist, B. Manil, and B. A. Huber, Phys. Rev. Lett. 97, 133401 (2006).

[18] S. Ptasińska, S. Denifl, V. Grill, T. D. Märk, E. Illenberger, and P. Scheier, Phys. Rev. Lett. 95, 093201 (2005).

[19] E. C. Montenegro, M. B. Shah, H. Luna, S. W. J. Scully, A. L. F. de Barros, J. A. Wyer, and J. Lecointre, Phys. Rev. Lett. 99, 213201 (2007).

[20] H. Levola, K. Kooser, E. Rachlew, E. Nõmmiste, and E. Kukk, Int. J. Mass Spectrom. 353, 7 (2013).

[21] T. Bergen, X. Biquard, A. Brenac, F. Chandezon, B. A. Huber, D. Jalabert, H. Lebius, M. Maurel, E. Monnand, J. Opitz, A. Pesnelle, B. Pras, C. Ristori, and J. C. Rocco, Rev. Sci. Instrum. 70, 3244 (1999).

[22] O. Plekan, M. Coreno, V. Feyer, A. Moise, R. Richter, M. de Simone, R. Sankari, and K. C. Prince, Phys. Scr. 78, 058105 (2008).

[23] See Supplemental Material at http://link.aps.org/ supplemental/10.1103/PhysRevLett.117.073201 for experimental details, fitting method, and list of electron densities of orbitals.

[24] E. Itälä, M. A. Huels, E. Rachlew, K. Kooser, T. Hägerth, and E. Kukk, J. Phys. B 46, 215102 (2013).

[25] Z. Deng, I. Bald, E. Illenberger, and M. A. Huels, Phys. Rev. Lett. 95, 153201 (2005).

[26] S. Ptasińska, P. Candori, S. Denifl, S. Yoon, V. Grill, P. Scheier, and T. Märk, Chem. Phys. Lett. 409, 270 (2005).

[27] G. Martinet, S. Díaz-Tendero, M. Chabot, K. Wohrer, S. Della Negra, F. Mezdari, H. Hamrita, P. Désesquelles, A. L. Padellec, D. Gardés, L. Lavergne, G. Lalu, X. Grave, J. F. 
Clavelin, P.-A. Hervieux, M. Alcamí, and F. Martín, Phys. Rev. Lett. 93, 063401 (2004).

[28] P. Bolognesi, J. A. Kettunen, A. Cartoni, R. Richter, S. Tosic, S. Maclot, P. Rousseau, R. Delaunay, and L. Avaldi, Phys. Chem. Chem. Phys. 17, 24063 (2015).

[29] L. S. Cederbaum, J. Phys. B 8, 290 (1975).

[30] M. J. Frisch et al., Gaussian 09 Revision C.01 (Gaussian Inc., Wallingford, CT, 2009).

[31] D. L. Yeager, Applied Many-Body Methods in Spectroscopy and Electronic Structure, edited by D. Mukherjee (Springer, New York, 1992), pp. 133-161.

[32] M. A. Hervé Du Penhoat, P. López-Tarifa, K. K. Ghose, Y. Jeanvoine, M. P. Gaigeot, R. Vuilleumier, M. F. Politis, and
M. C. Bacchus-Montabonel, J. Mol. Model. 20, 2221 (2014).

[33] A. Rentenier, L. F. Ruiz, S. Díaz-Tendero, B. Zarour, P. Moretto-Capelle, D. Bordenave-Montesquieu, A. Bordenave-Montesquieu, P. A. Hervieux, M. Alcamí, M. F. Politis, J. Hanssen, and F. Martín, Phys. Rev. Lett. 100, 183401 (2008).

[34] A. Bárány, G. Astner, H. Cederquist, H. Danared, S. Huldt, P. Hvelplund, A. Johnson, H. Knudsen, L. Liljeby, and K.-G. Rensfelt, Nucl. Instrum. Methods Phys. Res., Sect. B 9, 397 (1985).

[35] S. Díaz-Tendero, L. F. Ruiz, B. Zarour, J. Hanssen, M. Alcamí, M. F. Politis, P.-A. Hervieux, and F. Martín, J. Phys. Conf. Ser. 194, 012047 (2009). 